\title{
SY13-1
}

Symposium

\section{Research Partnaring Models of Bayer}

\section{Shunichi Takahashi}

\author{
Open Innovation Center, Bayer Yakuhin, Ltd., Japan
}

The process of drug development covers a wide range of activities from target identifications of drug discovery to clinical trials. The process requires specialized knowledge and highly developed skills. For many complex diseases the molecular mechanisms behind the disease are not well understood. In this environment the need for partnering of pharmaceutical companies with all other relevant stakeholders is getting more important. Pharmaceutical companies have developed a variety of partnering models. In this session, specifically focusing on the drug discovery, the various partnering models used by Bayer are to be discussed. 